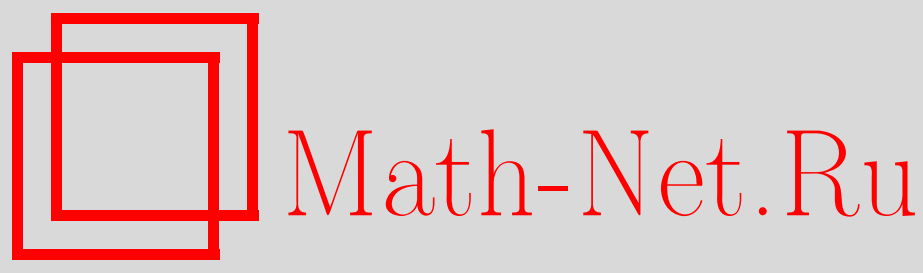

Е. Е. Тареева, Сферические аналоги некоторых моделей неизинговых спиновых стекол. Точные решения, ТМФ, 2011, том 168, номер 3, 584-592

DOI: https://doi.org/10.4213/tmf6702

Использование Общероссийского математического портала Math-Net.Ru подразумевает, что вы прочитали и согласны с пользовательским соглашением http://www . mathnet.ru/rus/agreement

Параметры загрузки:

IP : 54.210 .77 .194

26 апреля 2023 г., 12:21:49

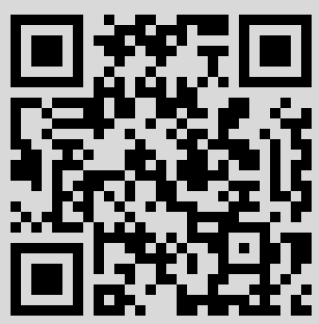




\title{
СФЕРИЧЕСКИЕ АНАЛОГИ НЕКОТОРЫХ МОДЕЛЕЙ НЕИЗИНГОВЫХ СПИНОВЫХ СТЕКОЛ. ТОЧНЫЕ РЕШЕНИЯ
}

\begin{abstract}
Рассмотрен ряд моделей неизинговых спиновых стекол, для которых удается естественным образом сформулировать аналог сферических условий, хорошо известных для модели Изинга. Статистические суммы полученных моделей вычисляются точно. Метод случайных матриц и метод реплик дают тождественные результаты.
\end{abstract}

Ключевые слова: спиновые стекла, сферическое приближение, точное решение.

В настоящей работе рассматриваются непрерывные аналоги некоторых известных дискретных неизинговых моделей спинового стекла, а именно спинового стекла спина единица, квадрупольного стекла и спиновых стекол Поттса с произвольным числом состояний. В этих моделях оказывается возможным ввести естественным образом условие, подобное хорошо известному сферическому условию для непрерывного аналога модели Изинга [1], и в итоге решить эти новые "сферические" модели точно. Существенным результатом является тот факт, что полученные с помощью теории случайных матриц точные результаты для этих моделей совпадают с полученными в работе результатами применения к этим моделям метода реплик.

Хотя, как хорошо известно, сферические модели являются весьма нереалистическими с физической точки зрения, они тем не менее имеют определенную ценность, так как представляют собой редкие примеры многочастичных систем, для которых статистическая сумма может быть вычислена точно.

Впервые сферическое приближение было сформулировано в работе Берлина и Каца [1], в которой дискретные изинговы спины с $S_{i}^{2}=1$ были заменены непрерывными, удовлетворяющими глобальному соотношению $\sum_{i} S_{i}^{2}=N$, где сумма берется по всем $N$ узлам решетки. Полученная задача была решена точно для взаимодействия ближайших соседей на различных трехмерных решетках.

Вскоре после появления первых теоретических работ по спиновым стеклам был предложен сферический вариант модели Шеррингтона-Киркпатрика [2], т. е. модели Изинга с бесконечным радиусом случайных взаимодействий, и получено его точное решение [3]. Вообще модели спиновых стекол с мягкими спинами довольно популярны в кинетическом подходе к спиновым стеклам, так как наличие одноузельных

* Институт физики высоких давлений РАН, Троицк, Московская обл., Россия. E-mail: etare@ms2.inr.ac.ru 
членов позволяет описать динамическое поведение стекол. Наиболее известной является сферическая модель $p$-спинового стекла квазиизинговых спинов (см., например, работы [4], [5]), поведение которой дает сценарий перехода жидкость-стекло.

В нашей работе [6] была предложена точно решаемая модель спинового стекла Поттса с тремя состояниями при дополнительных условиях, обобщающих сферические условия для изинговых спинов. В работе было получено точное решение с помощью теории случайных матриц. Было показано также, что метод реплик дает такое же решение. Полученное решение является репликосимметричным, устойчивым, так что нарушения репличной симметрии не требуется. Это отличает сферический вариант от собственно модели Поттса с тремя состояниями, для которой репликосимметричное решение устойчиво лишь в конечной области температур вблизи фазового перехода в состояние стекла [7], [8].

Настоящая работа может рассматриваться как прямое продолжение работы [6] в двух направлениях. Во-первых, будут рассмотрены сферические модели спинового стекла Поттса с произвольным числом состояний. Во-вторых, в модели, рассмотренной в работе [6], будет нарушена симметрия Поттса, что даст возможность исследовать сферические аналоги аксиального квадрупольного стекла и спинового стекла спина 1.

Модель спинового стекла Поттса задается гамильтонианом следующего вида:

$$
H=-\frac{p}{2} \sum_{i \neq j} J_{i j} \delta_{\sigma_{i}, \sigma_{j}},
$$

где $p$ - число состояний, а переменные $\sigma_{i}, \sigma_{j}$, заданные на узлах решетки $i, j$, могут принимать значения $0,1, \ldots, p-1 ; \delta_{\alpha \beta}-$ символ Кронекера. Таким образом, пара $\left\{\sigma_{i}, \sigma_{j}\right\}$ дает в энергию вклад $-J_{i j}$, если $\sigma_{i}=\sigma_{j}$, и не дает никакого вклада в противном случае. Связи $J_{i j}$ представляют случайные взаимодействия, распределенные по Гаyccy:

$$
P\left(J_{i j}\right)=\frac{\sqrt{N}}{\sqrt{2 \pi} \tilde{J}} e^{-\left(J_{i j}\right)^{2} N /\left(2 \tilde{J}^{2}\right)} .
$$

Множитель $N$ обеспечивает корректный термодинамический предел и среднеполевой характер модели. Спиновое стекло Поттса может служить моделью для ориентационного стекла в смешанных молекулярных кристаллах, а также в стеклах кластеров, когда сильная анизотропия окружения ограничивает возможные ориентации молекулы или кластера $p$ различными направлениями.

Обычно используется следующее представление модели Поттса (см., например, обзор [9]):

$$
H=-\frac{1}{2} \sum_{a=1}^{p-1} J_{i j} \sum_{i \neq j} J_{i j} \phi_{i}^{a} \phi_{j}^{a} .
$$

Здесь $(a, b, c, \ldots)$ обозначают $p-1$ спиновую компоненту, а $(i, j)$ - узлы решетки. Величины $\phi_{i}^{a}$ называются поттсовскими переменными и выбираются из множества $p$ векторов $\left\{e^{l}\right\}$, где $l=1,2, \ldots, p$, в $(p-1)$-мерном векторном пространстве,

$$
e_{a}^{l}=\left(\frac{p-a}{p+1-a}\right)^{1 / 2} p^{1 / 2} \psi,
$$


где $\psi=0$, если $l<a, \psi=1$, если $l=a$ и $\psi=-1 /(p-1)$, если $l>a$, при этом

$$
\sum_{l=1}^{p} e_{a}^{l} e_{b}^{l}=p \delta_{a b}, \quad \sum_{a=1}^{p-1} e_{a}^{l} e_{a}^{l^{\prime}}=p \delta_{l l^{\prime}}-1, \quad \sum_{l=1}^{p} e_{a}^{l}=0 .
$$

Могут использоваться и другие представления модели Поттса. Так, в частности, в работе [6] мы использовали предложенное нами ранее представление модели Поттса с тремя состояниями с помощью операторов квадрупольного момента для $\mathbf{J}=1$ : $Q=3 J_{z}^{2}-2, V=\sqrt{3}\left(J_{x}^{2}-J_{y}^{2}\right), J_{z}=1,0,-1$. Операторы $Q$ и $V$ коммутируют и в представлении, в котором они оба диагональны, имеют вид

$$
Q=\left(\begin{array}{ccc}
-2 & 0 & 0 \\
0 & 1 & 0 \\
0 & 0 & 1
\end{array}\right), \quad V=\left(\begin{array}{ccc}
0 & 0 & 0 \\
0 & \sqrt{3} & 0 \\
0 & 0 & -\sqrt{3}
\end{array}\right)
$$

Ниже для диагональных матриц мы будем использовать сокращенные обозначения типа $Q_{k}=(-2,1,1)$ и $V_{k}=(0, \sqrt{3},-\sqrt{3})$. Справедливо соотношение

$$
\frac{1}{6}\left(Q_{m(i)} Q_{n(j)}+V_{m(i)} V_{n(j)}+2\right)=\delta_{m n},
$$

обеспечивающее для гамильтониана

$$
H=-\frac{1}{2} \sum_{i \neq j} J_{i j}\left(Q_{i} Q_{j}+V_{i} V_{j}\right)
$$

эквивалентность гамильтониану модели Поттса с тремя состояниями.

Это квадрупольное представление оказалось полезным при рассмотрении ряда задач для модели Поттса с тремя состояниями, в частности при вычислении числа метастабильных состояний при нулевой температуре [10], при определении границы устойчивости $1 R S B$ решения в области низких температур [8], при определении разложений параметров порядка вблизи температуры перехода в стекло [11] и др. Дело в том, что в нашем подходе число интегрирований меньше, чем в стандартных, что дает целый ряд технических преимуществ.

Рассмотрим теперь модель Поттса с четырьмя состояниями $(p=4)$. Мы снова можем обратиться к операторам квадрупольного момента, теперь для $\mathbf{J}=3 / 2$. Выберем $Q^{\prime} \sim 3 J_{z}{ }^{2}-J(J+1), V^{\prime} \sim J_{z}$ и определим третий неединичный оператор $P^{\prime}$ так, чтобы он был ортогонгален операторам $Q^{\prime}$ и $V^{\prime}$. А именно, положим (см. работу [11]) $Q_{k}^{\prime}=(1,-1,-1,1), \sqrt{5} V_{k}^{\prime}=(-3,1,-1,3), \sqrt{5} P_{k}^{\prime}=(1,3,-3,-1)$. Теперь аналогичное (5) условие принимает вид

$$
Q_{m(i)}^{\prime} Q_{n(j)}^{\prime}+V_{m(i)}^{\prime} V_{n(j)}^{\prime}+P_{m(i)}^{\prime} P_{n(j)}^{\prime}+1=4 \delta_{m n},
$$

так что мы можем для модели Поттса с четырьмя состояниями использовать гамильтониан ${ }^{1)}$

$$
H=-\frac{1}{2} \sum_{i \neq j} J_{i j}\left[Q_{i}^{\prime} Q_{j}^{\prime}+V_{i}^{\prime} V_{j}^{\prime}+P_{i}^{\prime} P_{j}^{\prime}\right]
$$

1) Следует заметить, что наше представление с помощью трех матриц не является единственным. Например, можно использовать набор $A_{k}=(1,-1,-1,1), B_{k}=(-1,1,-1,1), C_{k}=(1,1,-1,-1)$. Мы используем, однако, приведенные выше операторы, чтобы, с одной стороны, сохранить физический смысл обобщенных квадрупольных моделей, а с другой стороны, рассматривать единым образом случаи четных и нечетных $p$. 
и ввести естественным образом аналог сферического условия в виде

$$
\sum_{i}\left(\left(Q_{i}^{\prime}\right)^{2}+\left(V_{i}^{\prime}\right)^{2}+\left(P_{i}^{\prime}\right)^{2}\right)=3 N
$$

Диагонализуем прежде всего матрицу $J_{i j}$ с помощью ортогонального преобразования. При этом билинейная форма $\sum_{i \neq j} J_{i j} x_{i} x_{j}$ преобразуется в $\sum_{\lambda} J_{\lambda} x_{\lambda}^{2}$, а переменные $Q_{i}^{\prime}, V_{i}^{\prime}$ и $P_{i}^{\prime}$ переходят в новые переменные $Q_{\lambda}^{\prime}, V_{\lambda}^{\prime}$ и $P_{\lambda}^{\prime}$, определяемые соотношениями

$$
Q_{\lambda}^{\prime}=\sum_{i}\langle\lambda \mid i\rangle Q_{i}^{\prime}, \quad V_{\lambda}^{\prime}=\sum_{i}\langle\lambda \mid i\rangle V_{i}^{\prime}, \quad P_{\lambda}^{\prime}=\sum_{i}\langle\lambda \mid i\rangle P_{i}^{\prime},
$$

где $\langle\lambda|$ представляет ортонормированный собственный вектор матрицы $J_{i j}$, принадлежащий собственному значению $J_{\lambda}$. В пределе $N \rightarrow \infty$ плотность собственных значений $\rho\left(J_{\lambda}\right)$ подчиняется закону (см., например, монографию [12])

$$
\rho\left(J_{\lambda}\right)=\frac{1}{2 \pi \tilde{J}^{2}}\left(4 \tilde{J}^{2}-J_{\lambda}^{2}\right)^{1 / 2}
$$

и суммы $(1 / N) \sum_{\lambda} f\left(J_{\lambda}\right)$ могут быть заменены интегралами $\int d J \rho(J) f(J)$, где $\rho(J)$ дается формулой (11).

Ограничение (9) на интегрирование в статистической сумме может быть учтено с помошью соответствующей $\delta$-функции. Используя интегральное представление для $\delta$-функции и проведя интегрирование по переменным $Q_{\lambda}^{\prime}, V_{\lambda}^{\prime}$ и $P_{\lambda}^{\prime}$, как это обычно делается при рассмотрении сферических моделей, получаем для статистической суммы (с точностью до несущественного множителя) выражение

$$
Z=\int_{a-i \infty}^{a+i \infty} \frac{d z}{2 \pi i} \exp \left\{N\left[3 z-\frac{3}{2 N} \sum_{\lambda} \ln \left(z-\frac{J_{\lambda}}{2 T}\right)\right]\right\},
$$

где контур интегрирования лежит правее наибольшего собственного значения $2 \tilde{J}$. Далее будем опускать штрихи у переменных $Q^{\prime}, V^{\prime}$ и $P^{\prime}$.

При получении выражения (12) мы использовали соотношение

$$
\int \ldots \int \exp \left(-\sum_{i, j=1}^{N} a_{i j} x_{i} x_{j}\right) \prod d x_{i}=\pi^{N / 2}\left(\operatorname{det}\left|a_{i j}\right|\right)^{-1 / 2} .
$$

Уравнение для седловой точки имеет вид

$$
1-\frac{1}{4 \pi \tilde{J}^{2}} \int_{-2 \tilde{J}}^{2 \tilde{J}} d J \frac{\sqrt{(2 \tilde{J})^{2}-J^{2}}}{z-J /(2 T)}=0
$$

или

$$
z-\sqrt{z^{2}-t^{2}}=t^{2}
$$

где $t=\tilde{J} / T$. Уравнение (15) имеет решение $z=\left(1+t^{2}\right) / 2$, если $T>T_{\mathrm{c}}$, где $T_{\mathrm{c}}$

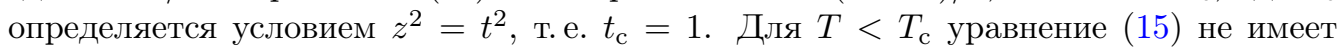
действительного решения, и седловая точка $z$ соответствует "прилипанию" к $t$, точке ветвления подынтегрального выражения в формуле (12) (см. подробнее в работе [3]). 
Статистической сумме (12) соответствует свободная энергия на узел, усредненная по спектру $\rho(J)$, вида $\left(k_{\mathrm{B}}=1\right)$

$$
\begin{aligned}
\langle f(T)\rangle_{a v} & =-\frac{3 T}{2}-\frac{3 \tilde{J}^{2}}{4 T}-\frac{3 T}{2} \ln 2, & & T>T_{\mathrm{c}}, \\
\langle f(T)\rangle_{a v} & =-3 \tilde{J}+\frac{3 T}{4}+\frac{3 T}{2} \ln \frac{\tilde{J}}{2 T}, & & T<T_{\mathrm{c}} .
\end{aligned}
$$

Нетрудно посчитать и остальные термодинамические величины.

Найдем теперь свободную энергию для гамильтониана (8) с помощью метода реплик. Действуя стандартным образом (см., например, работы [2], [6]), получим для свободной энергии на узел следующее выражение:

$$
\begin{aligned}
-\beta f & =\lim _{N \rightarrow \infty} \lim _{n \rightarrow 0} \frac{1}{n N}\left\{\int _ { a - i \infty } ^ { a + i \infty } d z \left[\int d Q_{1} \ldots d Q_{n} d V_{1} \ldots d V_{n} d P_{1} \ldots d P_{n} d y d x \times\right.\right. \\
& \times \exp \left(-\frac{3 n(n-1) y^{2}}{4}-\frac{3 n x^{2}}{2}+3 n z-z \sum_{\alpha}\left(Q_{\alpha}^{2}+V_{\alpha}^{2}+P_{\alpha}^{2}\right)\right) \times \\
& \left.\left.\times \exp \left(\frac{t y}{2} \sum_{\alpha \neq \beta}\left(Q_{\alpha} Q_{\beta}+V_{\alpha} V_{\beta}+P_{\alpha} P_{\beta}\right)+\frac{t x}{\sqrt{2}} \sum_{\alpha}\left(Q_{\alpha}^{2}+V_{\alpha}^{2}+P_{\alpha}^{2}\right)\right)\right]^{N}-1\right\} .
\end{aligned}
$$

Здесь $x \sim\left\langle Q_{\alpha}^{2}\right\rangle=\left\langle V_{\alpha}^{2}\right\rangle=\left\langle P_{\alpha}^{2}\right\rangle, y \sim\left\langle Q_{\alpha} Q_{\beta}\right\rangle=\left\langle V_{\alpha} V_{\beta}\right\rangle=\left\langle P_{\alpha} P_{\beta}\right\rangle$.

$\mathrm{B}$ репликосимметричном приближении свободная энергия принимает вид

$$
-\beta f=\frac{3 t^{2}}{4}+3 z-\frac{3 x^{2}}{2}+\frac{3 y^{2}}{4}-\frac{3}{2} \ln \left(z-\frac{x t}{\sqrt{2}}+\frac{y t}{2}\right)+\frac{3 t y}{4(z-x t / \sqrt{2}+y t / 2)} .
$$

Здесь переменные $x, y$ и $z$ удовлетворяют условиям экстремума:

$$
\begin{gathered}
4\left(z-\frac{x t}{\sqrt{2}}+\frac{y t}{2}\right)^{2}-2\left(z-\frac{x t}{\sqrt{2}}+\frac{y t}{2}\right)-t y=0, \\
\frac{t^{2} y}{4(z-x t / \sqrt{2}+y t / 2)^{2}}=y, \\
\frac{t}{2 \sqrt{2}(z-x t / \sqrt{2}+y t / 2)}+\frac{t^{2} y}{4 \sqrt{2}(z-x t / \sqrt{2}+y t / 2)^{2}}=x .
\end{gathered}
$$

Решение уравнения (20) дает

$$
z-\frac{x t}{\sqrt{2}}+\frac{y t}{2} \rightarrow \frac{1}{2}
$$

при $y \rightarrow 0$. Таким образом, из уравнения (21) получаем критическую температуру (точку ветвления) $t_{\mathrm{c}}^{2}=1$.

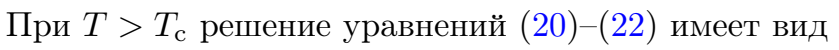

$$
y=0, \quad x=\frac{t}{\sqrt{2}}, \quad z=\frac{1}{2}\left(1+t^{2}\right),
$$


и свободная энергия в репликосимметричном приближении (19) совпадает с (16).

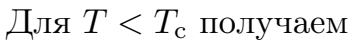

$$
y=t-1, \quad x=\frac{t}{\sqrt{2}}, \quad z=t
$$

так что (19) совпадает с выражением (17). Следуя работе [3], легко показать, что поведение параметров порядка одинаково в обоих подходах. Для стандартного стекольного параметра порядка $q$ имеем $q=y / t=1-1 / t$.

Таким образом, репликосимметричное решение для модели Поттса с четырьмя состояниями совпадает с точным решением, полученным выше. Хочется обратить внимание на то, что снова мы имеем ситуацию, аналогичную сферическому стеклу Шеррингтона-Киркпатрика [3], а не p-спиновому сферическому стеклу [5], хотя исходная дискретная модель Поттса с четырьмя состояниями не обладает отражательной симметрией. Суть здесь заключается в том, что в случае двухчастичных взаимодействий использование непрерывных переменных эффективно восстанавливает отражательную симметрию. Это легко понять, построив гамильтониан Гинзбурга-Ландау при использовании преобразования Хаббарда-Стратановича подобно тому, как это было сделано в работе [13]. Действительно, в дискретном случае ряды для $\operatorname{Tr}_{A} e^{\phi A}$ содержат нечетные степени величины $\phi$, если $\operatorname{Tr} A^{2 n+1} \neq 0$, в то время как в непрерывном случае соответствующие интегралы всегда равны нулю, что приводит к отсутствию таких членов. Некоторый “след” от кубических членов, приводящих к внутренним полям, сохранился в наличии переменной $x$. Для сохранения отсутствия отражательной симметрии в непрерывном случае необходимо, чтобы члены с нечетными степенями входили в условие мягкости. Например, для модели Поттса с тремя состояниями надо добавить к гамильтониану выражение

$$
\delta H=\sum_{i}\left(\left(Q_{i}^{2}+V_{i}^{2}\right)^{2}+\frac{4}{3}\left(Q_{i}^{3}-3 Q_{i} V_{i}^{2}\right)-4\left(Q_{i}^{2}+V_{i}^{2}\right)\right) .
$$

Заметим, что рассмотрение, подобное приведенному выше для модели Поттса с четырьмя состояниями, можно провести и для любого числа состояний $p$. Действительно, всегда можно найти набор из $p-1$ матрицы $A_{1}, A_{2}, \ldots, A_{p-1}$, который будет удовлетворять условию

$$
\sum_{k=1}^{p-1} A_{k, \alpha} A_{k, \beta}+B=C \delta_{\alpha \beta}
$$

что является следствием условий (4). При этом, если выбрать нормировку матриц таким образом, чтобы $C-B=p-1$, то результаты будут в точности совпадать с результатами для случая изинговых спинов, т. е. свободная энергия для сферического стекла Поттса с $p$ состояниями $F_{p}^{\mathrm{sph}}$ будет иметь вид $F_{p}^{\mathrm{sph}}=(p-1) F_{\mathrm{SK}}^{\mathrm{sph}}$, где $F_{\mathrm{SK}}^{\mathrm{sph}}-$ свободная энергия для сферического варианта модели Шеррингтона-Киркпатрика [3].

Вернемся теперь к модели Поттса с тремя состояниями. Нарушим поттсовскую симметрию в гамильтониане (6), т. е. пусть

$$
H=-\frac{1}{2} \sum_{i \neq j}\left(J_{i j} Q_{i} Q_{j}+G_{i j} V_{i} V_{j}\right)
$$


где $G_{i j}$ - некоторое отличное от $J_{i j}$ гауссово распределение. По-прежнему справедливо сферическое условие в виде

$$
\sum_{i}\left(Q_{i}^{2}+V_{i}^{2}\right)=4 N
$$

Теперь статистическая сумма примет вид

$$
Z=\int_{a-i \infty}^{a+i \infty} \frac{d z}{2 \pi i} \exp \left\{N\left[4 z-\frac{1}{2 N} \sum_{\lambda} \ln \left(z-\frac{J_{\lambda}}{2 T}\right)-\frac{1}{2 N} \sum_{\lambda} \ln \left(z-\frac{G_{\lambda}}{2 T}\right)\right]\right\}
$$

и условие для седловой точки запишется следующим образом:

$$
4-\frac{T^{2}}{\tilde{J}^{2}}\left(z-\sqrt{z^{2}-\frac{\tilde{J}^{2}}{T^{2}}}\right)-\frac{T^{2}}{\widetilde{G}^{2}}\left(z-\sqrt{z^{2}-\frac{\widetilde{G}^{2}}{T^{2}}}\right)=0 .
$$

Таким образом, в полной аналогии с изложенным ранее мы можем рассмотреть сферический аналог анизотропной квадрупольной модели, исходный дискретный вариант которой был детально исследован в работе [14]. Чтобы излишне не загромождать изложение, рассмотрим предельные случаи, когда $J_{i j}=0$ или $G_{i j}=0$. Поскольку операторы $Q$ и $V$ входят в гамильтониан и сферическое условие симметричным образом, то безразлично, что полагать равным нулю: получающиеся формулы идентичны. При $\tilde{J}=0$ физический смысл задачи - аксиальное квадрупольное стекло [15] в сферическом приближении, а при $\widetilde{G}=0$ имеем сферическую модель спинового стекла спина 1 , так как $V=\sqrt{3} S_{z}$ (см. выше).

Из выражения (26) получим при $G_{\lambda}=0$ свободную энергию в седловой точке:

$$
-\beta f=4 z-\frac{z^{2}}{2 t^{2}}+\frac{z \sqrt{z^{2}-t^{2}}}{2 t^{2}}-\frac{1}{2} \ln \left(z+\sqrt{z^{2}-t^{2}}\right)-\frac{1}{2} \ln z+\frac{1}{2} \ln 2+\frac{1}{4},
$$

и уравнение для седловой точки

$$
4-\frac{z}{t^{2}}+\frac{\sqrt{z^{2}-t^{2}}}{t^{2}}=\frac{1}{2 z}=0
$$

которое можно записать в виде уравнения третьей степени

$$
-32 z^{3}+8 z^{2}\left(8 t^{2}+1\right)-16 z t^{2}+t^{2}=0 .
$$

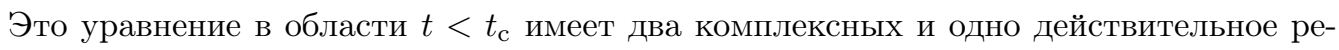
шение. Мы не приводим их из-за громоздкости формул. При $z=t$ интересующее нас действительное решение "прилипает" (см. выше), что и определяет температуру перехода. Проще всего ее определить, решая уравнение (30) относительно $t$ при $z=t$. Это дает $t_{\mathrm{c}}=3 / 8$.

С помощью метода реплик нетрудно получить для свободной энергии сферической квадрупольной модели в репликосимметричном приближении следующее выражение:

$$
-\beta f=4 z-\frac{x^{2}}{2}+\frac{y^{2}}{4}-\frac{1}{2} \ln z-\frac{1}{2} \ln \left(z-\frac{x t}{\sqrt{2}}+\frac{y t}{2}\right)+\frac{t y}{4(z-x t / \sqrt{2}+y t / 2)} .
$$


Здесь переменные $x, y$ и $z$ удовлетворяют условиям экстремума. В частности, уравнение, определяющее появление ненулевого решения для параметра порядка стекла, совпадает с уравнением (21), а решение уравнения, аналогичного (20), дает

$$
z=\frac{t}{8 t-2}
$$

при $y \rightarrow 0$. Таким образом, из уравнения (21) получаем критическую температуру (точку ветвления) $t_{\mathrm{c}}=3 / 8$. При температурах ниже точки перехода, когда $t>3 / 8$, условия экстремума принимают вид

$$
\begin{gathered}
4-\frac{1}{t}-\frac{y}{t}-\frac{1}{2 z}=0, \\
2 z-x t \sqrt{2}+y t-t=0, \\
-x \sqrt{2}+1+y=0 .
\end{gathered}
$$

При подстановке решения этих уравнений

$$
z=t, \quad x=\frac{\sqrt{2}}{4}(8 t-1), \quad y=\frac{1}{2}(8 t-3)
$$

в свободную энергию (31) получим, что при $T<T_{\mathrm{c}}(t=\tilde{J} /(k T))$

$$
-\beta f=4 t-\ln t+\frac{1}{2} \ln 2-\frac{1}{4} .
$$

Такое же выражение получается из уравнения (28) при $z=t$.

Таким образом, во всех рассмотренных моделях обобщенных спиновых стекол удалось естественным образом ввести условия типа сферических, которые позволили получить точное решение с помощью теории случайных матриц. Эти же модели были исследованы с помощью метода реплик. Во всех случаях результаты точных решений совпали с результатами метода реплик в репликосимметричном приближении.

Благодарности. Автор благодарит Т. И. Щелкачеву и В. Н. Рыжова за полезные обсуждения. Работа поддержана РФФИ (грант № 11-02-00341), а также федеральной целевой программой "Научные и научно-педагогические кадры инновационной России” на 2009-2013 гг. (контракт № 02.740.11.0432).

\section{Список литературы}

[1] T. H. Berlin, M. Kac, Phys. Rev., 86:6 (1952), 821-835.

[2] D. Sherrington, S. Kirkpatrick, Phys. Rev. Lett., 35:26 (1975), 1792-1796.

[3] R. Jones, J. Kosterlitz, D. Thouless, Phys. Rev. Lett., 36:20 (1976), 1217-1220.

[4] T. R. Kirkpatrick, P. G. Wolynes, Phys. Rev. B, 36:16 (1987), 8552-8564; T. R. Kirkpatrick, D. Thirumalai, Phys. Rev. B, 37:10 (1988), 5342-5350; D. Thirumalai, T. Kirkpatrick, Phys. Rev. B, 38:7 (1988), 4881-4892.

[5] A. Crisanti, H. J. Sommers, Z. Phys. B, 87:3 (1992), 341-354.

[6] N. V. Gribova, V. N. Ryzhov, E. E. Tareyeva, Phys. Lett. A, 353:2003 (2006), 226-229.

[7] D. J. Gross, I. Kanter, H. Sompolinsky, Phys. Rev. Lett., 55:3 (1985), 304-307. 
[8] N. V. Gribova, V.N. Ryzhov, E. E. Tareyeva, Phys. Rev. E, 68:6 (2003), 067103, 4 pp., arXiv: cond-mat/0307042.

[9] F. Y. Wu, Rev. Modern Phys., 54:1 (1982), 235-268.

[10] E. A. Lutchinskaia, E. E. Tareyeva, Europhys. Lett., 17:2 (1992), 109-112.

[11] N. V. Gribova, T. I. Schelkacheva, E. E. Tareyeva, J. Phys. A, 43:49 (2010), 495006, 9 pp., arXiv: 1007.0135 .

[12] M. L. Mehta, Random Matrices and the Statistical Theory of Energy Levels, Academic Press, New York, 1967.

[13] N. V. Gribova, V. N. Ryzhov, T. I. Schelkacheva, E. E. Tareyeva, Phys. Lett. A, 315:6 (2003), 467-473, arXiv: cond-mat/0211700.

[14] Е. А. Лучинская, Е. Е. Тареева, ТМФ, 70:3 (1987), 477-480.

[15] Е. А. Лучинская, В. Н. Рыжов, Е. Е. Тареева, ТМФ, 67:3 (1986), 463-469; E. A. Lutchinskaia, V. N. Ryzhov, E. E. Tareyeva, J. Phys. C, 17:26 (1984), L665-L667.

Поступила в редакцию 3.03.2011 